\title{
Efficacy of Praziquantel Treatment for Schistosoma mansoni Infection Among Children in Ethiopia: Systematic Review and Meta-analysis
}

\author{
Habtye Bisetegn", Yonas Erkihun \\ Department of Medical Laboratory Science, Wollo University, Dessie, Ethiopia \\ Email address: \\ habtiye21@gmail.com (H. Bisetegn),yonas.erkihun@wu.edu.et (Y. Erkihun) \\ ${ }^{*}$ Corresponding author
}

\section{To cite this article:}

Habtye Bisetegn, Yonas Erkihun. Efficacy of Praziquantel Treatment for Schistosoma mansoni Infection Among Children in Ethiopia: Systematic Review and Meta-analysis. International Journal of Immunology. Vol. 8, No. 4, 2020, pp. 61-69.

doi: $10.11648 /$ j.iji.20200804.11

Received: October 24, 2020; Accepted: November 7, 2020; Published: November 16, 2020

\begin{abstract}
Background: Schistosomiasis is a chronic neglected tropical disease of poverty caused by blood dwelling trematodes of Schistosoma species. Praziquantel (PZQ) is the current drug of choice for the treatment of human schistosomiasis. The existence of Praziquantel resistance by Schistosoma species is a threat and an alarm for strict monitoring and periodic evaluation of its efficacy. Method: We searched PubMed, PubMed Center, Google Scholar, Web of Science, Google, Science Direct, MEDLINE and proceedings of a professional association to identify studies published in English and assessed the efficacy of Praziquantel against Schistosoma mansoni infection in children. Studies reporting the efficacy of Praziquantel in both adult and children as study participants and studies which investigated the efficacy of Praziquantel combined with other antischistosomal drugs were excluded. Result: Since there was significant heterogeneity, the random effect model was used. A total of 9 papers involving 1,412 participants, that assessed the cure rate and 8 papers that assessed egg reduction rate of Praziquantel were included in the meta-analysis. The pooled cure rate of Praziquantel was $86.65 \%$ with $95 \%$ CI; 83.4-93.9. There was high heterogeneity $\left(I^{2}=93.4 \%\right)$. Subgroup analysis showed slightly higher Cure rate of Praziquantel in Amhara regional state compared to Oromia regional state $(88.58 \%$ vs $84.79 \%)$. The egg reduction rate was $87.95 \%$ and $99.85 \%$ using geometric and arithmetic mean of egg count respectively. The prevalence of Schistosoma mansoni decreased from $55.77 \%$ to $11.29 \%$ after administration of Praziquantel. Conclusion: According to the result of this review, standard dose of Praziquantel was effective against Schistosoma mansoni infection among children in Ethiopia. The cure rate of Praziquantel was slightly higher in Amhara regional state compared to Oromia regional state. The drug showed a great reduction in egg count and intensity of infection.
\end{abstract}

Keywords: Praziquantel, Schistosoma mansoni, Efficacy, Ethiopia

\section{Introduction}

Schistosomiasis is a chronic neglected tropical disease of poverty caused by blood dwelling digenetic trematodes of the Schistosoma species. The disease is responsible for a major public health problem and imposed a socioeconomic burden in developing countries. It is endemic in 78 countries, of which most of them are resource-limited [1]. It is a serious public health problem with 200 up to 209 million people being infected. Globally approximately 779 million people are living in an area with active transmission of schistosomiasis [2]. In 2016 about 190 million people were already infected with schistosomiasis [3]. In 2016 About 237.2 million people in 51 countries require preventive chemotherapy for schistosomiasis [4]. Clinically schistosomiasis can have different forms that can be varied according to endemicity and immune response. The manifestation can begin with cutaneous rash, hypersensitivity reactions, abdominal pain, dry cough, general fatigue, myalgia, diarrhea, 
abdominal tender and cardiac and central nervous involvement may rarely occurred at sever stage of the infection [5]. Poor sanitations like open field defecation, improper latrine utilization, poor waste management and poor personal hygiene are major concerns in developing countries. Children in those countries spent most of their time by swimming or bathing in contaminated water, thus increasing the chance of acquiring schistosomiasis [6]. Praziquantel (PZQ) is a pyrazinoquinoline derivative that is recommended by WHO as preventive chemotherapy for schistosomiasis with a $40 \mathrm{mg} / \mathrm{kg}$ standard dose. Due to its high efficacy and safety PZQ replace other antischistosomal drugs, and it leads to a great reduction in schistosomiasis and soil transmitted helminthes [7]. Different factors such as species of the parasite, stage of the parasite, and intensity of infection affect the therapeutic efficacy of Praziquantel for treating schistosomiasis [8]. Praziquantel can be given at different does and can be administered as a single or repeated dose. A repeated standard dose of a $40 \mathrm{mg} / \mathrm{kg}$ PZQ was reported to have achieved enhanced efficacy compared to a single dose [9]. Praziquantel at standard dose is fairly effective for treating Schistosoma mansoni with cure rate (CR) ranging from $79.4 \%$ to $88.6 \%$ [9]. In schistosomiasis endemic countries, PZQ mass chemotherapy has been implemented as part of school based or community based campaign in order to control morbidity associated with schistosomiasis and to reduce its transmission [10-13]. Scholars had reported the presence of Schistosoma mansoni resistance to PZQ mainly in endemic areas such as Africa [7]. Studies in Egypt and Senegal had reported the resistance of Schistosoma mansoni infection to PZQ $[14,15]$. The existence of drug resistance of Schistosoma species to PZQ is a threat. It is also alarm for adequate monitoring during mass drug administration and periodic evaluation of its efficacy. It is also indication for developing new alternative drugs.

Ethiopia is among schistosomiasis high burden countries with 37.3 million people living at risk of infection; of this about 3.4 million and 12.3 million are pre-school and school age children respectively [16]. In Ethiopia Schistosomiasis is a major public health problem. It occurs in two clinical forms; intestinal schistosomiasis that is caused by Schistosoma mansoni and urogenital schistosomiasis by Schistosoma hematobium. Schistosoma mansoni is endemic in Ethiopia with pooled prevalence of $18.3 \%$ in the total population [17]. Ethiopia had a long term plan to eliminate schistosomiasis related morbidity by 2020 and to reduce the prevalence of heavy infection below $1 \%$. The country had also planned to treat 8.9 million school age children for schistosomiasis by $2015 / 16$ and had treated 6.66 million school age children this indicate $74.8 \%$ achievement [16]. Even though the achievement is good the prevalence is still higher in school children. Assessing the drug efficacy, reinfection rate and selecting the most effective drug is mandatory to eliminate the disease associated morbidity as well as to reduce the prevalence of the disease below $1 \%$. Therefore this systematic review provides the overall (pooled) efficacy of Praziquantel for treating Schistosoma mansoni infection among preschool and school age children at country level (Ethiopia).

\section{Method}

\subsection{Literature Search}

The search period for this study was from July 1 to September 28, 2020. Systematic searching of literatures (published, unpublished and ongoing research) was carried out by using systematic electronic search on PubMed, PubMed center, Google scholar, Web of Science, Google, Science Direct, MEDLINE. Besides proceedings of professional association like Ethiopian medical laboratory association and Ethiopian public health association were searched. Searching of citation of the searched articles was also carried out. The search was conducted using key words and MESH terms; efficacy, Praziquantel, schistosomiasis, Schistosoma mansoni, school age children, pre-school children, children and Ethiopia.

\subsection{Inclusion and Exclusion}

Studies that reported the cure rate and/or egg reduction rate of Praziquantel for Schistosoma mansoni infection were included in this meta-analysis. Studies in which Praziquantel was administered at $40 \mathrm{mg} / \mathrm{kg}$ in a single standard dose and involving pre-school and school age children were also included in this meta-analysis. Studies conducted in a language other than English language were excluded. Studies that reported efficacy of Praziquantel in both adult and children as study participants; studies that reported efficacy of Praziquantel combined with other anti schistosomal drugs; studies that assessed efficacy of Praziquantel in schistosomiasis and other coinfection as well as studies that assessed efficacy of Praziquantel against Schistosoma hematobium were also excluded.

\subsection{Data Extraction and Quality Assessment}

The authors (Habtye B. and Yonas E.) screened the title, abstract and full text of all identified literatures and identified potentially eligible studies. All included studies were added to Excel and extracted by Habtye B. then crosschecked by Yonas E. Missing or unclear outcome data were asked from the investigators. Study characteristics and variables like cure rate, egg reduction rate, study method, sample size; study site; mean or median age, gender, study group, region of origin and publication year of the study were extracted from eligible studies by using Microsoft excel. The primary out come this review and meta-analysis was the cure rate (CR) and egg reduction rate (ERR) of PZQ at a dose of $40 \mathrm{mg} / \mathrm{kg}$ for Schistosoma mansoni infection treatment.

The two reviewers independently assessed the quality of the searched studies included in the review using Jaded scoring criteria [18]. The quality score was cross-checked and differences were resolved by rechecking the quality score 
by the two investigators together and through discussion.

\subsection{Data Analysis}

Statistical analysis of this meta-analysis was conducted by using STATA version 12. The efficacy of Praziquantel was assessed in terms of cure rate $\mathrm{CR}$ and egg reduction rate (ERR). Cure rate was defined as the proportion of children who are negative to Schistosoma mansoni after treatment given but who were positive at baseline to the number of positive children before treatment. The egg reduction rate was defined as the proportional reduction in the mean eggs per gram of post treatment vs. pretreatment calculated using either geometric or arithmetic mean and reported separately. The pooled $\mathrm{CR}$ and the pooled ERR were calculated and reported using forest plot. The confidence interval for both out comes (CR and ERR) was set at $95 \%(95 \% \mathrm{CI})$. Since there was heterogeneity of efficacy estimate from the studies, the summary outcome (CR and ERR) was calculated by using Random effect meta-analysis with inverse-variance weight [19]. The heterogeneity of the studies was assessed using $I^{2}$ statistics as measured by Higgins and Thompson [20, 21]. $I^{2}$ statistics is the reliable and preferred test for measuring heterogeneity across studies. $I^{2}$ value $<25 \%$ indicate homogeneity of studies while values $25 \%-75 \%$ and $\geq 75 \%$ indicate moderate and very high heterogeneity across studies [20]. Publication bias was assessed by visual inspection of funnel plot and eggers test statistic with $p$-value $\leq 0.05$ indicating the presence of publication bias [22, 23].

\subsection{Ethical Consideration}

This systematic review and meta-analysis was conducted in accordance with the PRISMA guidelines [24]. This study is systematic review and meta-analysis, therefore ethical permission is not required.

\section{Result}

\subsection{Literature Search Result and Characteristics of Included Studies}

In this systematic review and meta-analysis, a total of 276 articles were found from systematic literature search. After screening the title, abstract and full text of the searched literatures, 13 studies met the eligibility criteria. Four studies were excluded as the two studies assessed PQZ efficacy in schistosomiasis and helminths co-infection and two other studies assessed PZQ against Schistosoma hematobium with different study participants. Finally 9 studies were included in the meta-analysis (Figure 1). Of the included studies eight of them also assessed the ERR of PZQ and reported using geometric and arithmetic mean. The included studies were conducted between 2002 and 2020. The included studies were conducted in four Ethiopian national regional states where schistosomiasis is known to be endemic. Eight of them assessed PZQ efficacy among school age children and one study use pre-school children as study participant. The studies involve 1,412 children as study participants. In all studies, the standard dose given to each child was $40 \mathrm{mg} / \mathrm{kg}$. The post treatment assessment time was 3-6 weeks later. Four of the included studies performed post-treatment assessment after 4 weeks of the drug administration while two studies assessed after 3 weeks. One study assessed post treatment efficacy after 6 month and two studies did not indicate the post treatment assessment time. All studies used Kato-Katz thick smear as diagnostic method. The estimated cure rate of PZQ ranges from $73.60 \%-99.10 \%$. There was no uniform reporting method for the ERR. Five studies used geometric mean to report ERR and reported ERR as $68.2 \%$ $99.5 \%$, while two studies used arithmetic mean and reported 99.5\% and 99.9\% ERR (Table 1).

Table 1. Summary of characteristics of included studies.

\begin{tabular}{|c|c|c|c|c|c|c|}
\hline Author/s/Reference & publication year & study area /region/ & Sample size & Reporting methods for ERR & CR (\%) & $\operatorname{ERR}(\%)$ \\
\hline Tadesse Dejenie, et al [25] & 2010 & Tigray regional state & 225 & NA & 91.11 & NA \\
\hline Makida Kemal, et al [26] & 2019 & Somali Regional state & 59 & Geometric mean & 96.4 & NA \\
\hline Mitiku Bajiro, et al [27] & 2016 & Oromia Regional State & 120 & Arithmetic mean & 99.1 & 99.4 \\
\hline Getinet Degu, et al [28] & 2002 & Amhara Regional state & 148 & NA & 94 & 99.9 \\
\hline Birehanu Reta, et al [29] & 2013 & Amhara Regional state & 187 & Geometric mean & 82.89 & 97 \\
\hline Birehanu Erko, et al [30] & 2012 & Oromia Regional State & 144 & Geometric mean & 73.6 & 79.46 \\
\hline Samuel Haile, et al [31] & 2012 & Oromia Regional State & 204 & Geometric & 80.9 & 68.2 \\
\hline Eden Woldegerima, et al [32] & 2019 & Amhara Regional state & 80 & Arithmetic mean & 90 & 99.51 \\
\hline Addisu Tesfie, et al [33] & 2020 & Amhara Regional state & 245 & Geometric mean & 86.9 & 99.5 \\
\hline
\end{tabular}

$\mathrm{NA}=$ not available; $\mathrm{CR}=$ cure rate; $\mathrm{ERR}=$ egg reduction rate 


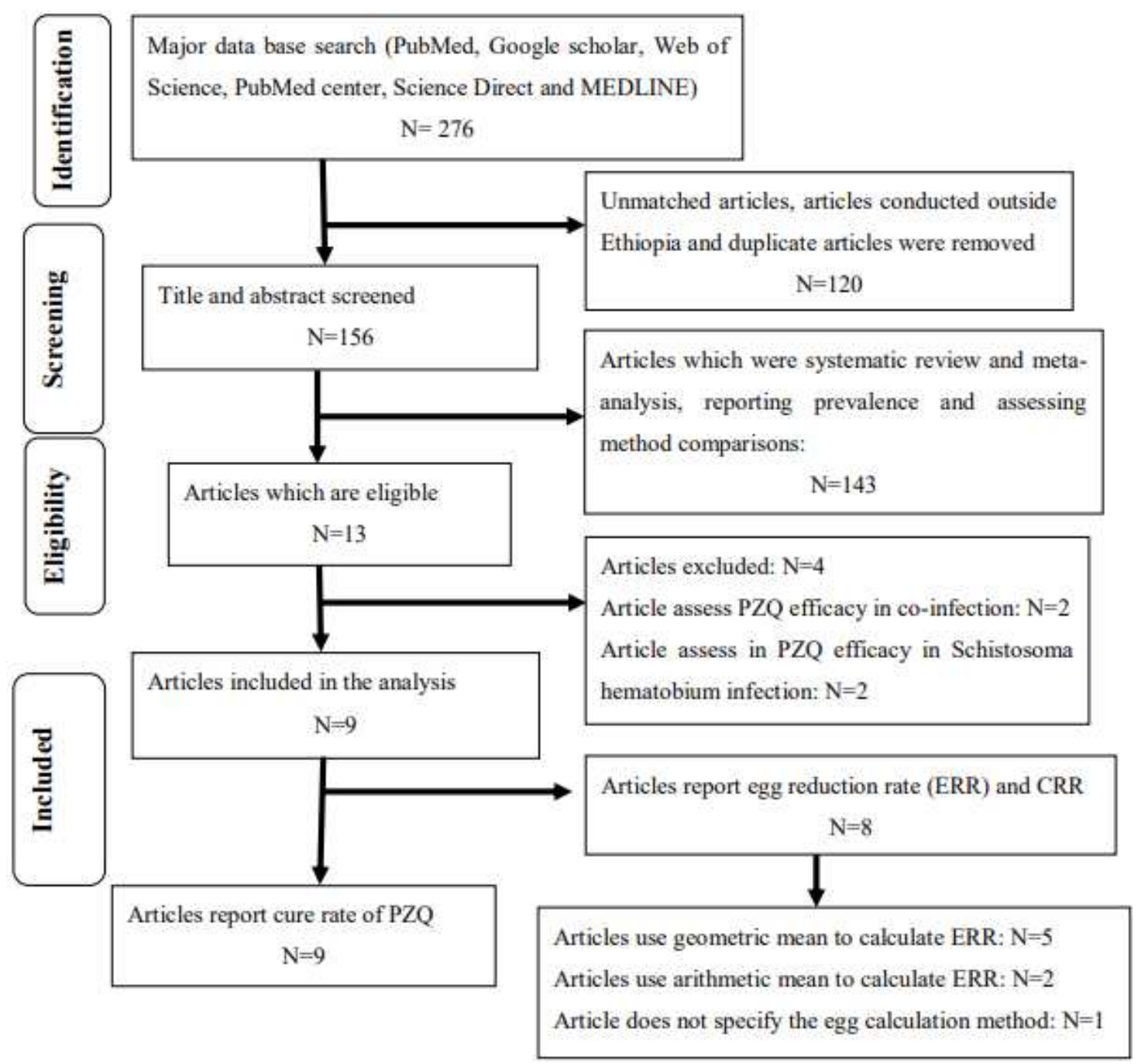

Figure 1. Flow diagram showing article selection process.

The authors also assessed the intensity of infection and the pre/post prevalence of Schistosoma mansoni infection. According to the reviewed articles, the intensity of infection in most of the study participants were low ranging from $22.52 \%-70 \%$. But in one study the participants were in a status moderate and heavy infection only. The drug had reduced the prevalence of Schistosoma mansoni infection among children from (24-74.9\%) to (3.39\%-26.3\%) (Table 2).

Table 2. Pre and post treatment prevalence of Schistosomk a mansoni and intensity of infection

\begin{tabular}{lllll}
\hline \multirow{2}{*}{ Author/s/ } & \multirow{2}{*}{ Pre/post treatment prevalence (\%) } & \multicolumn{2}{l}{ Intensity of infection (\%) } & High \\
\cline { 3 - 5 } & & Low & Medium & 10.57 \\
Tadesse Dejenie, et al & $67.95 / 8.66$ & 50.94 & 50.49 & 0 \\
Makida Kemal, et al & $25 / 3.39$ & 40.70 & $30 \%$ & 20 \\
Mitiku Bajiro, et al & $24.00 / 0.90$ & 70 & NA & NA \\
Getinet Degu, et al & $50.80 / 6.08$ & NA & 22.52 & 35.59 \\
Birehanu Reta, et al & $70.47 / 17.11$ & 26.30 & 42.40 \\
Birehanu Erko, et al & $74.90 / 26.30$ & 35.30 & 31.30 & $26 \%$ \\
Samuel Haile, et al & $67.60 / 19.12$ & 0 & 38.70 & 13.80 \\
Eden Woldegerima, et al & $35 / 10$ & 26.50 & 86.20 & 36.30 \\
Addisu Tesfie, et al & $83.30 / 13.10$ & & 37.30 & \\
\hline
\end{tabular}

Cure rate of Praziquantel against Schistosoma mansoni infection

\subsection{Meta-analysis of Cure rate of Praziquantel}

The pooled cure rate of PZQ against Schistosoma mansoni infection was $86.65 \%$ with $95 \%$ CI (83.4-93.9). There was high heterogeneity among studies with $I^{2}$ of $93.4 \%$ (figure 2 ). 


\begin{tabular}{|c|c|c|c|}
\hline Author/s & year & ES $(95 \% \mathrm{Cl})$ & $\begin{array}{l}\% \\
\text { Weight }\end{array}$ \\
\hline Tadesse Dejenie, et al & 2010 & $91.11(87.39,94.83)$ & 11.60 \\
\hline Makida Kemal, et al & 2019 & $96.40(91.65,101.15)$ & 11.18 \\
\hline Mitiku Bajiro, et al & 2016 & $99.10(97.41,100.79)$ & 12.16 \\
\hline Getinet Degu, et al & 2002 & $94.00(90.17,97.83)$ & 11.56 \\
\hline Birehanu Reta, et al & 2013 & - $82.89(77.49,88.29)$ & 10.89 \\
\hline Birehanu Erko, et al & 2012 & $73.60(66.40,80.80)$ & 10.00 \\
\hline Samuel Haile, et al & 2012 & $80.90(75.51,86.29)$ & 10.90 \\
\hline Eden Woldegerima, et al & 2019 & — $90.00(83.43,96.57)$ & 10.32 \\
\hline Addisu Tesfie, et al & 2020 & $86.90(82.68,91.12)$ & 11.40 \\
\hline \multicolumn{2}{|c|}{ Overall (I-squared $=93.4 \%, p=0.000)$} & $88.65(83.40,93.90)$ & 100.00 \\
\hline NOTE: Weights are from rz & fects analysis & & \\
\hline
\end{tabular}

Figure 2. Forest plot showing pooled efficacy (cure rate) of 40mg/kg PZQ against Schistosoma mansoni infection among pre-school and school age children.

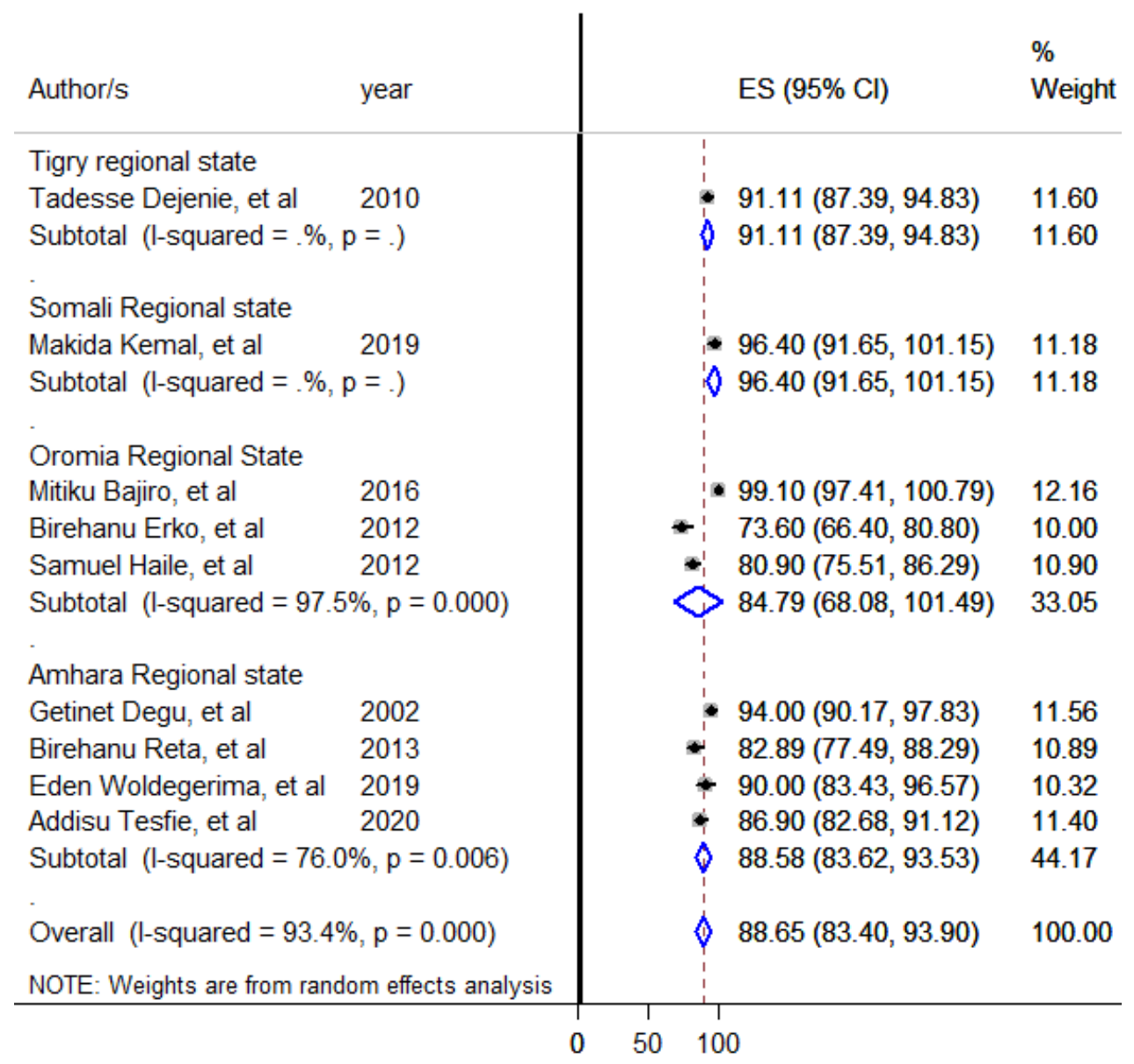

Figure 3. Forest plot showing subgroup analysis of cure rate of PZQ by the different regional states of Ethiopia. 


\subsection{Subgroup Analysis}

Since there was substantial heterogeneity, subgroup analysis was done by the region where the studies were conducted. The articles were from four different regional state of Ethiopia. But subgroup analysis was done for only two regional states. The remaining two regional states had only one published article for each as a result meta-analysis cannot be done. The overall cure rate of PZQ was higher in Amhara regional state compared to the cure rate in Oromia regional state. The pooled cure rates were $88.58 \%$ and $84.79 \%$ in Amhara and Oromia regional state respectively. Heterogeneity was also high after subgroup analysis. It was $97.5 \%$ in Oromia regional state but, in Amhara regional state the heterogeneity was moderate with $I^{2}$ value of $76 \%$ (figure 3 ).

\subsection{Publication Bias}

Publication bias was assessed by visual inspection of the

\begin{tabular}{|c|c|c|c|}
\hline Author/s & $\begin{array}{l}\text { publication } \\
\text { year }\end{array}$ & $\mathrm{ES}(95 \% \mathrm{Cl})$ & $\begin{array}{l}\% \\
\text { Weight }\end{array}$ \\
\hline \multicolumn{4}{|l|}{ Geometric mean } \\
\hline Makida Kemal, et al & 2019 & $99.40(97.43,101.37)$ & 14.21 \\
\hline Getinet Degu, et al & 2002 & $97.00(94.25,99.75)$ & 13.44 \\
\hline Birehanu Reta, et al & 2013 & $\begin{array}{ll}- & 79.46(73.67,85.25)\end{array}$ & 9.68 \\
\hline Birehanu Erko, et al & 2012 & $\begin{array}{r}-\quad 68.20(60.59,75.81) \\
\end{array}$ & 7.68 \\
\hline Samuel Haile, et al & 2012 & • $99.51(98.55,100.47)$ & 14.91 \\
\hline Addisu Tesfie, et al & 2020 & $+78.30(73.14,83.46)$ & 10.46 \\
\hline \multicolumn{2}{|c|}{ Subtotal $($ l-squared $=97.0 \%, p=0.000)$} & $087.95(81.69,94.21)$ & 70.38 \\
\hline \multicolumn{4}{|c|}{. } \\
\hline \multicolumn{4}{|l|}{ Arithmetic mean } \\
\hline Mitiku Bajiro, et al & 2016 & $99.90(99.33,100.47)$ & 15.06 \\
\hline Eden Woldegerima, et al & 2019 & $99.50(97.95,101.05)$ & 14.56 \\
\hline \multicolumn{2}{|c|}{ Subtotal $(I-$ squared $=0.0 \%, p=0.634)$} & $99.85(99.32,100.38)$ & 29.62 \\
\hline & & \\
\hline \multicolumn{2}{|c|}{ Overall $($ l-squared $=96.1 \%, p=0.000)$} & $92.65(89.65,95.65)$ & 100.00 \\
\hline \multicolumn{2}{|c|}{ NOTE: Weights are from random effects analysis } & 1 & \\
\hline
\end{tabular}

Figure 4. Forest plot showing egg reduction rate of PZQ by the two reporting methods.

Pre-treatment and post treatment prevalence of Schistosoma mansoni

According to the reviewed articles the pooled prevalence Schistosoma mansoni before administration of standard dose of $40 \mathrm{mg} / \mathrm{kg}$ PZQ was $55.77 \%$. After administration of the drug the prevalence reduced to $11.28 \%$ (Figure 5). symmetry of the funnel plot and by the egger's test statistics. The funnel plot does not show symmetry (Figure 4) and eggers test statistics showed the presence of publication bias with p-value of 0.002 . Therefore, trim and fill meta-analysis has been done to in order to account for the publication bias. The pooled cure rate of PZQ was found to be 86.63 which is similar to the frist one.

\subsection{Meta-analysis of Egg Reduction Rate (ERR)}

Due to high heterogeneity, random effect meta-analysis was done to assess the pooled ERR of PZQ against Schistosoma mansoni infection. Reporting the ERR of PZQ was different depending on the method used to calculate ERR. The pooled ERR by using geometric mean was $87.95 \%$ $(95 \% \mathrm{CI} ; 81.69 \%, 94.21 \%)$ with high heterogeneity $\left(\mathrm{I}^{2}=97 \%\right)$. The pooled ERR by using arithmetic mean it was $99.85 \%$ $(95 \% \mathrm{CI} ; 99.32 \%, 100.38 \%)$ with no heterogeneity.

68

68

0.38 


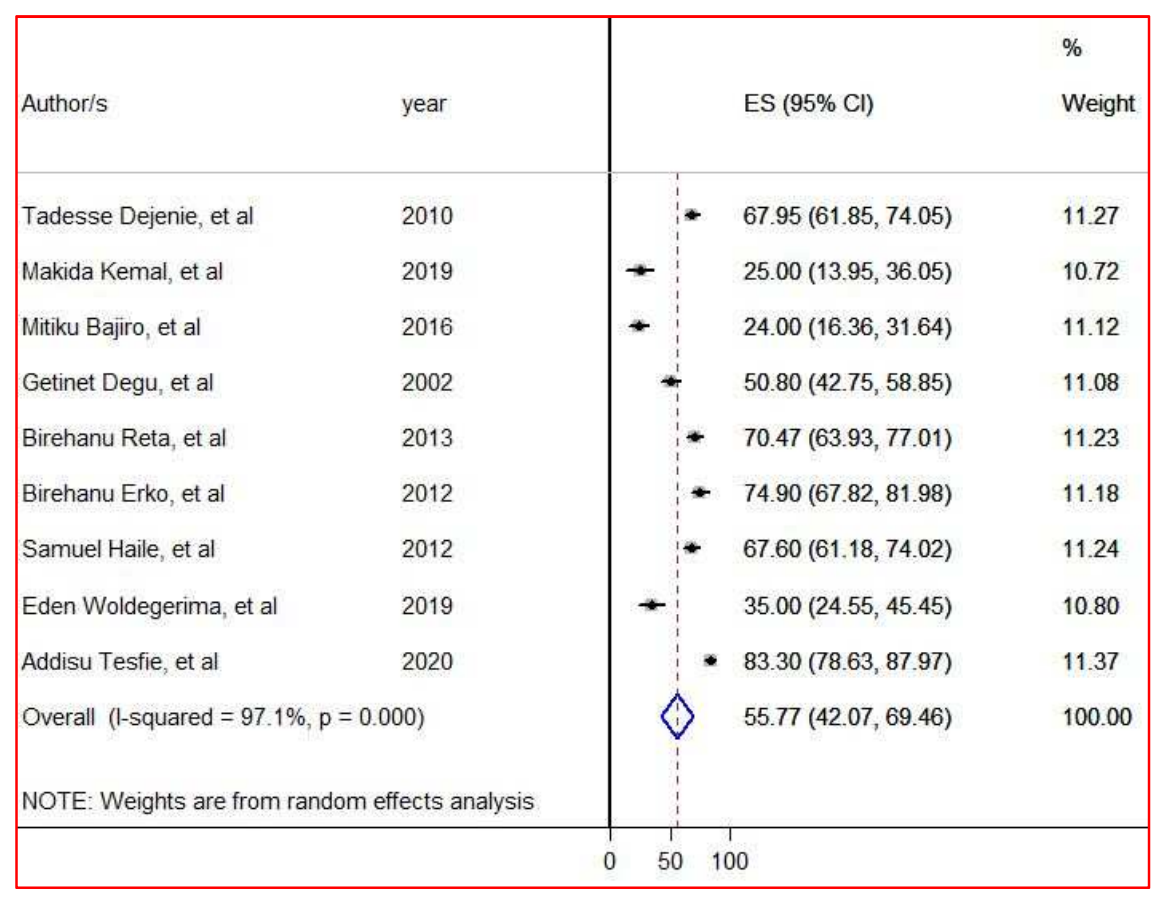

\begin{tabular}{|ll|ll|}
\hline Author/s & year & ES $(95 \%$ Cl) & Weight \\
\hline Tadesse Dejenie, et al & 2010 & $8.66(4.99,12.33)$ & 11.61 \\
Makida Kemal, et al & 2019 & $3.39(-1.23,8.01)$ & 11.23 \\
Mitiku Bajiro, et al & 2016 & $0.90(-0.79,2.59)$ & 12.16 \\
Getinet Degu, et al & 2002 & $6.08(2.23,9.93)$ & 11.54 \\
Birehanu Reta, et al & 2013 & $17.11(11.71,22.51)$ & 10.88 \\
Birehanu Erko, et al & 2012 & $26.30(19.11,33.49)$ & 9.99 \\
Samuel Haile, et al & 2012 & $19.12(13.72,24.52)$ & 10.88 \\
Eden Woldegerima, et al & 2019 & $10.00(3.43,16.57)$ & 10.31 \\
Addisu Tesfie, et al & 2020 & $13.10(8.88,17.32)$ & 11.40 \\
Overall (l-squared $=93.3 \%, \mathrm{p}=0.000)$ & $11.29(6.07,16.52)$ & 100.00 \\
& & & \\
\hline NOTE: Weights are from random effects analysis & & & \\
\hline
\end{tabular}

Figure 5. Forest plot showing the pooled prevalence of Schistosoma mansoni during pre-PZQ treatment in the left panel and post-PZQ treatment.

\section{Discussion}

Praziquantel is antihelminthic pyrazinoisoquinoline derivatives discovered in 1972 and developed in 1975 as a broad spectrum drug against helminthic Cestodes and trematodes by E. Merck and Bayer AG, Germany [34]. It is the WHO recommended antischistosomal drug that causes death of the parasite by rapidly contracting the worm muscle and leading to lose of worm movement, bleb formation and vacuolization of tegument which later on results in rupture of the vacuole and blebs. It exhibit stage specific killing mechanism [9]. A standard dose of $40 \mathrm{mg} / \mathrm{kg}$ PZQ showed a good protective efficacy for both pre-school and school age children with CR of $72 \%(95 \%$ CI; 54.8-85.8 ) and 69\% (95\% CI; 53.4-81.8) respectively [35].

This systematic review was conducted in order to estimate the pooled cure rate and egg reduction rate of PZQ against Schistosoma mansoni infection among pre-school and school age children from 2002 [28] up to 2020 [33]. In this systematic review and meta-analysis, the cure rate of PZQ against Schistosoma mansoni infection among children ranges from $73.6 \%$ to $99.1 \%$ with an overall pooled CR of $86.65 \%$ with $95 \%$ CI of $83.4 \%$ - $93.9 \%$. In this review, the $\mathrm{CR}$ is at the top and slightly better than previously reported reviews $63 \%-85 \%$ by Wegner et al [36]. According to our 
review a single dose $40 \mathrm{mg} / \mathrm{kg}$ PZQ give excellent protection against acute schistosomiasis and its protection rate is higher than review and meta-analysis done by Liu $\mathrm{R}$ et al, which reported $52 \%$ (95\% CI: $49 \%-55 \%$ ) protective rate [37]. The difference in efficacy may due to difference period of assessment between the first doses and follow up, the stage of the parasite present in the host, immune status of the participants. The drug effectively reduces the prevalence of the diseases from $55.77 \%$ to $11.29 \%$.

Subgroup analysis was also done for the two national regional states of Ethiopia. The pooled cure rate was higher Amhara regional state as compared to Oromia regional state ( $88.58 \%$ vs $84.79 \%)$. Significant heterogeneity was observed even after performing subgroup analysis.

Egg reduction rate is also currently recommended as another method of assessing efficacy of drug [38]. In the reviewed articles, researchers used either geometric mean or arithmetic mean of the egg count. Meta-analysis of articles that used geometric mean to report ERR showed a $87.95 \%$ (95\%CI; 81.69\%, 94.21\%) pooled ERR of PZQ against Schistosoma mansoni infection, while in those that used arithmetic mean of egg count the pooled ERR was $99.85 \%$ (95\%CI; 99.32\%, 100.38\%). Praziquantel showed significant egg reduction rate indicating promising protective capacity against Schistosoma mansoni infection. Its high cure rate and egg reduction rate found in this meta-analysis can prove the great role of PZQ to achieve the goal of controlling schistosomiasis associated morbidity by the year 2020 and to eliminate schistosomiasis from the public health problem by the year 2025 set by the WHO [39].

\section{Conclusion}

This systematic review and meta-analysis showed PZQ administered at standard dose $(40 \mathrm{mg} / \mathrm{kg})$ had promising efficacy against Schistosoma mansoni infection among children in Ethiopia. The review indicated treatment of Schistosoma mansoni infection with PZQ will result in high CR and ERR. The cure rate of PZQ was higher in Amhara national regional state than Oromia national regional state. The drug also showed high reduction in egg count and intensity of infection. The drug showed variable efficacy with great variation from study to study and to area this indicates the need for periodic evaluation of the drug at regional as well as national level in order to achieve the goal of eliminating morbidity and mortality of children associated with schistosomiasis.

\section{Abbreviations}

CR: Cure Rate; ERR: Egg Reduction Rate; $P Z Q$ : Praziquantel; WHO: World Health Organization.

\section{Funding Source}

There was no specific funding for this systematic review and meta-analysis.

\section{Availability of Data and Materials}

The datasets analyzed during the current study are available from the corresponding author upon reasonable request.

\section{Author's Contribution}

Habtye B. and Yonas E. searched, analyzed and interpreted the data. Habtye B. and Yonas E. wrote the first draft of the manuscript. Habtye B. revised the manuscript. All authors read and approved the final version of the manuscript.

\section{Declaration of Interest}

The authors declared that they have no competing interest.

\section{Acknowledgements}

The authors would like to acknowledge all authors of studies included in this systematic review and meta-analysis.

\section{References}

[1] Karunamoorthi K, Almalki M, Ghailan K. Schistosomiasis: A neglected tropical disease of poverty: A call for intersectoral mitigation strategies for better health. J Health Res Rev. 2018; 5 (1): 1 .

[2] WHO. WHO Weekly epidemiological record. 2012; 87 (4): $37-44$.

[3] World Health Organization, Department of Control of Neglected Tropical Diseases. Integrating neglected tropical diseases into global health and development: fourth WHO report on neglected tropical diseases. 2017.

[4] WHO. Preventive chemotherapy and transmission control databank for schistosomiasis. Available from: available at http://www.who.int/neglected_diseases/preventive_chemother apy/sch/en/index.html

[5] Stefanie Knopp, So“ren L Becker, Katrin J Ingram, Jennifer Keiser, Ju"rg Utzinger. Diagnosis and treatment of schistosomiasis in children in the era of intensified control. Expert Rev Anti Infect Ther. 2013; Available from: www.expert-reviews.com

[6] CDC. Parasite. Schistosomiasis. 2012; Available from: http://www.cdc.gov/parasites/ schistosomiasis/

[7] Wang W, Wang L, Liang Y-S. Susceptibility or resistance of praziquantel in human schistosomiasis: a review. Parasitol Res. 2012; 111 (5): 1871-7.

[8] King CH, Olbrych SK, Soon M, Singer ME, Carter J, Colley DG. Utility of Repeated Praziquantel Dosing in the Treatment of Schistosomiasis in High-Risk Communities in Africa: A Systematic Review. Keiser J, editor. PLoS Negl Trop Dis. 2011; 5 (9): e1321.

[9] Muhubiri Kabuyaya, Moses John Chimbari, Samson Mukaratirwa. Efficacy of praziquantel treatment regimens in pre-school and school aged children infected with schistosomiasis in subSaharan Africa: a systematic review. Infect Dis Poverty. 2018; 7: 73. 
[10] Chai J-Y. Praziquantel Treatment in Trematode and Cestode Infections: An Update. Infect Chemother. 2013; 45 (1): 32.

[11] M. G. Chipeta, B. Ngwira, L. N. Kazembe. Analysis of schistosomiasis haematobium infection prevalence and intensity in Chikhwawa, Malawi: an application of a two part mode. PLoS Negl Trop Dis. 2013; 7 (3): e2131.

[12] L. Savioli, A. F. Gabrielli, A. Montresor, L. Chitsulo, D. Engels. Schistosomiasis control in Africa: 8 years after World Health Assembly Resolution 54·19. Parasitology. 2009; 136 (13): 1677-1681

[13] WHO. Preventive Chemotherapy in Human Helminthiasis: Coordinated Use of Anthelmintic Drugs in Control Interventions. Man Health Prof Programme Manag World Health Organ Geneva Switz. 2006;

[14] Fallon PG, Sturrock RF, Capron A, Niang M, Doenhoff MJ. Short Report: Diminished Susceptibility to Praziquantel in a Senegal Isolate of Schistosoma mansoni. Am J Trop Med Hyg. 1995; 53 (1): 61-2.

[15] Ismail M, Metwally A, Farghaly A, Bruce J, Tao L-F, Bennett JL. Characterization of Isolates of Schistosoma mansoni from Egyptian Villagers that Tolerate High Doses of Praziquantel. Am J Trop Med Hyg. 1996; 55 (2): 214-8.

[16] Nebiyu Negussu, Birhan Mengistu, Biruck Kebede, Kebede Deribe, Ephrem Ejigu, Gemechu Tadesse, et al. Ethiopia Schistosomiasis and Soil-Transmitted Helminthes Control Programme: Progress and Prospects. Ethiop Med J. 2017; 55 (1): 75-80.

[17] Hussen S, Assegu D, Shimelis T. Prevalence of Schistosoma mansoni infection in Ethiopia: A systematic review and metaanalysis. Microbiology; 2019. Available from: http://biorxiv.org/lookup/doi/10.1101/610113

[18] Jadad AR, Moore RA, Carroll D, Jenkinson C, Reynolds DJ, Gavaghan DJ, et al. Assessing the quality of reports of randomized clinical trials: is blinding necessary? Control Clin Trials. 1996; 17: 1-12.

[19] Egger M, Smith GD, Phillips AN. Meta-analysis: Principles and procedures. BMJ. 1997 Dec 6; 315 (7121): 1533-7.

[20] Higgins JPT, Thompson SG. Quantifying heterogeneity in a meta-analysis. Stat Med. 2002; 21 (11): 1539-58.

[21] Higgins JPT. Measuring inconsistency in meta-analyses. BMJ. 2003; 327 (7414): 557-60.

[22] Tang J-L, Liu JL. Misleading funnel plot for detection of bias in meta-analysis. J Clin Epidemiol. 2000; 53 (5): 477-84.

[23] Ried, K. Interpreting and understanding meta-analysis graphs: a practical guide. Aust Fam Physician. 2006; 35 (8): 635-8.

[24] Moher D, Liberati A, Tetzlaff J, Altman DG, PRISMA Group. Preferred reporting items for systematic reviews and metaanalyses: the PRISMA statement. Ann Intern Med. 2009; 151 (4): 264-9, W64.

[25] Tadesse Dejenie, Tsehaye Asmelash, Mahmud Abdelkadir. Efficacy of Praziquantel in Treating Schistosoma Mansoni Infected School Children in Tumuga and Waja, North Ethiopia. MEJS). 2010; 2 (2): 3-11.
[26] Kemal M, Tadesse G, Esmael A, Abay SM, Kebede T. Schistosoma mansoni infection among preschool age children attending Erer Health Center, Ethiopia and the response rate to praziquantel. BMC Res Notes. 2019 Dec; 12 (1): 211.

[27] Bajiro M, Dana D, Ayana M, Emana D, Mekonnen Z, Zawdie $B$, et al. Prevalence of Schistosoma mansoni infection and the therapeutic efficacy of praziquantel among school children in Manna District, Jimma Zone, southwest Ethiopia. Parasit Vectors. 2016; 9 (1): 560.

[28] Getinet Degu, Getahun Mengistu, Janet Jones. Praziquantel efficacy against schistosomiasis mansoni in schoolchildren in north-west Ethiopia. Trans R Soc Trop Med Hyg. 2002; 96: 444-5.

[29] Reta B, Erko B. Efficacy and side effects of praziquantel in the treatment for Schistosoma mansoni infection in school children in Senbete Town, northeastern Ethiopia. Trop Med Int Health. 2013; 18 (11): 1338-43.

[30] Erko B, Degarege A, Tadesse K, Mathiwos A, Legesse M. Efficacy and side effects of praziquantel in the treatment of Schistosomiasis mansoni in schoolchildren in Shesha Kekele Elementary School, Wondo Genet, Southern Ethiopia. Asian Pac J Trop Biomed. 2012; 2 (3): 235-9.

[31] Samuel Haile, * Lemu Golassa, Zeleke Mekonnen. Prevalence of Schistosoma mansoni and effectiveness of Praziquantel in school children in Finchaa valley, Ethiopia. J Parasitol Vector Biol. 2012; 4 (3): 25-30.

[32] Woldegerima E, Bayih AG, Tegegne Y, Aemero M, Jejaw Zeleke A. Prevalence and Reinfection Rates of Schistosoma mansoni and Praziquantel Efficacy against the Parasite among Primary School Children in Sanja Town, Northwest Ethiopia. J Parasitol Res. 2019; 2019: 1-8.

[33] Tesfie A, Getnet G, Abere A, Yihenew G, Belete Y, Kassa M, et al. Praziquantel is an effective drug for the treatment of Schistosoma Mansoni infection among school-aged children in Northwest Ethiopia. Trop Med Health. 2020; 48 (1): 28.

[34] Andrews P, Thomas H, Pohlke R, Seubert Jür. Praziquantel. Med Res Rev. 1983; 3 (2): 147-200.

[35] Jean T Coulibaly, Gordana Panic, Kigbafori D Silué, Jana Kovač, Jan Hattendorf, Jennifer Keiser. Efficacy and safety of praziquantel in preschool-aged and school-aged children infected with Schistosoma mansoni: a randomised controlled, parallel-group, dose-ranging, phase 2 trial. Lancet Glob Health. 2017; 5: e688-98.

[36] Wegner, D. H. G. The profile of the trematodicidal compound praziquante. Arzneimittelforschung. 1984; 34: 1132-1136.

[37] Liu R, Dong H-F, Guo Y, Zhao Q-P, Jiang M-S. Efficacy of praziquantel and artemisinin derivatives for the treatment and prevention of human schistosomiasis: a systematic review and meta-analysis. Parasit Vectors. 2011 Dec; 4 (1): 201.

[38] World Health Organization. Assessing the efficacy of anthelminthic drugs against schistosomiasis and soiltransmitted helminthiase. Geneva World Health Organ. 2013;

[39] World Health Organization (WHO). Schistosomiasis: Progress Report 2001-2011 and Strategic Plan 2012-2020. Geneva World Health Organ. 2012. 